\title{
Two-Frame Optical Flow Formulation in an Unwarping Multiresolution Scheme
}

\author{
C. Cassisa ${ }^{1,2}$, S. Simoens ${ }^{1}$, and V. Prinet ${ }^{2}$ \\ ${ }^{1}$ Lab. of Fluid Mechanics and Acoustics (LMFA), \\ Ecole Centrale Lyon, France \\ ${ }^{2}$ Lab. of Informatics, Automatics and Applied Mathematics (LIAMA), \\ Chinese Academy of Sciences, Beijing, Chine \\ ccassisa@ec-lyon.fr
}

\begin{abstract}
In this paper, we propose a new formulation of the Differential Optical Flow Equation (DOFE) between two consecutive images considering spatial and temporal information from both. The displacement field is computed in a Markov Random Field (MRF) framework. The solution is done by minimization of the Gibbs energy using a Direct Descent Energy (DDE) algorithm. A hybrid multiresolution approach, combining pyramidal decomposition and two-step multigrid techniques, is used to estimate small and large displacements. A new pyramidal decomposition method without warping process between pyramid levels is introduced. The experiments carried out on benchmark dataset sequences show the effectiveness of the new optical flow formulation using the proposed unwarped pyramid decomposition schema.
\end{abstract}

Keywords: Optical flow estimation, RMF minimization, Multiresolution technique.

\section{Introduction}

Motion estimation has always been a major activity in computer vision community, with application in tracking, stereo matching, rigid and elastic motions, fluid propagation... Since early 80's, it has been well studied and many approaches have been proposed. But it is still remaining challenging to this date. For details on existing algorithms, you can refer to Barron's et al. 44.

Differential Optical Flow Equation (DOFE), introduced by Horn \& Schunck [9, has proved to be very powerful in motion estimation. The DOFE is based on the hypothesis of illumination constancy over a small period of time. At the beginning, approaches were defined on a centered formulation of the DOFE that needs at least three successive images ([94]). Other approaches studied the case of only two successive frames and proposed a non-centered DOFE based on the first image ([5]) or on the second one ([11]3]). Recently the work of Alvarez et al. 1] imagines an intermediate image at the half way from the first to the second image and uses a symmetrical formulation of DOFE based on two images. However, this method needs many interpolation and warping steps that can affect the quality of the estimation.

E. Bayro-Corrochano and J.-O. Eklundh (Eds.): CIARP 2009, LNCS 5856, pp. 790-797, 2009.

(C) Springer-Verlag Berlin Heidelberg 2009 
The hypothesis of small displacement made to define DOFE is very restrictive. Most of movements do not respect this assumption over image domain. To deal with it, multiresolution techniques are commonly used. The idea is to estimate the displacement field in an incremental and iterative way for different image resolution (coarse-to-fine). The different resolutions can be generated by scale-space theory (2]) that convolves Gaussian filter of different variances to the image to extract coarse to fine information or by pyramidal decomposition of original images into successive images of smaller resolution. Many decomposition techniques were proposed as Gaussian pyramid [5]11]13], steerable pyramid [15] or wavelet decomposition [10. But all these approaches, during the multiresolution process, warp the image by the coarse displacement field estimated at upper pyramid level before computing the missing incremental displacement between the warped and the other image. This step transforms and interpolates image information. It is strongly correlated to the quality of coarse displacement field.

These last years, many works has been done on the search of the optimal solution of the displacement field. Due to the non-convexity of problem formulation, multigrid technique is often used ([12]). It allows local minimization to not be trapped in local minima. It has been shown that coupling multiresolution and multigrid techniques for optical flow estimation can improve the accuracy of the estimation (87]).

In the present work, we propose a new non-centered formulation of the DOFE that refers to the two image spatial information (TI_DOFE). The DOFE is solved by maximizing a posterior probability using a Direct Descent Energy (DDE) algorithm through the minimization of an equivalent MRF-Gibbs energy. Making a local spatial assumption, we define a multiresolution technique that does not need to warp image between two pyramid levels. As the previous work in [7], the multiresolution is combined with a two-step multigrid technique helping DDE to converge to the optimal solution while improving significantly the computational time.

The rest of the paper is organized as follows. Section 2 defines TI_DOFE, formulates the MRF framework and introduces the minimization method. In section 3 , we detail the pyramidal multiresolution schema using warping or unwarping steps and the combined multigrid technique. Results about three different sequences are illustrated and discussed in section 4 . Section 5 concludes the paper.

\section{Methodology}

\subsection{Two-Frame Optical Flow Equation}

For a two-frame temporal image sequence, the optical flow equation (OFE) is the $2 \mathrm{D}$ vector field of apparent displacement $\mathbf{d}(\mathbf{s})=(d x(\mathbf{s}), d y(\mathbf{s}))$ that links pixels $\mathbf{s}=(x, y)$ of the first image at time $t$ with its correspondent position in the second image at time $t+\Delta t$.

OFE definition is based on the assumption that the image illumination $(I(\mathbf{s}, t))$ is constant over a small time interval $\Delta t$ : 


$$
I(\mathbf{s}+\mathbf{d}(\mathbf{s}), t+\Delta t)-I(\mathbf{s}, t) \approx 0
$$

Making the hypothesis of a small displacement $\mathbf{d}(\mathbf{s})$ over a small time interval $\Delta t$, the Differential Optical Flow Equation (DOFE) can be computed from a $1^{\text {st }}$ order Taylor expansion of $I(\mathbf{s}+\mathbf{d}(\mathbf{s}), t+\Delta t)$ around $\mathbf{s}$ :

$$
I(\mathbf{s}+\mathbf{d}(\mathbf{s}), t+\Delta t)=I(\mathbf{s}, t+\Delta t)+\mathbf{d}(\mathbf{s}) \cdot \nabla I(\mathbf{s}, t+\Delta t)+\vartheta\left(\mathbf{d}^{2}(\mathbf{s})\right)
$$

If $\mathbf{d}(\mathbf{s})$ is small enough, $\vartheta\left(\mathbf{d}^{2}(\mathbf{s})\right)$ can be neglected. We have the following DOFE:

$$
I(\mathbf{s}, t+\Delta t)-I(\mathbf{s}, t)+\mathbf{d}(\mathbf{s}) \cdot \nabla I(\mathbf{s}, t+\Delta t) \approx 0
$$

With $\nabla I(\mathbf{s}, t+\Delta t)=\left(\frac{\partial I(x, y, t+\Delta t)}{\partial x}, \frac{\partial I(x, y, t+\Delta t)}{\partial y}\right)$ spatial gradients at time $t+\Delta t$ (second image). We call this equation DOFE_2.

Doing $1^{\text {st }}$ order Taylor expansion of $I(\mathbf{s}+\mathbf{d}(\mathbf{s}), t+\Delta t)$ around $\mathbf{s}$ and $\Delta t$. For $\mathbf{d}(\mathbf{s})$ and $\Delta t$ small enough, $\vartheta\left(\mathbf{d}^{2}(\mathbf{s}), \Delta t^{2}\right) \approx 0$ and DOFE can be rewrite as:

$$
\Delta t . I_{t}(\mathbf{s}, t)+\mathbf{d}(\mathbf{s}) \cdot \nabla I(\mathbf{s}, t) \approx 0
$$

With $I_{t}(\mathbf{s}, t)=\frac{\partial I(x, y, t)}{\partial t}$ and $\nabla I(\mathbf{s}, t)=\left(\frac{\partial I(x, y, t)}{\partial x}, \frac{\partial I(x, y, t)}{\partial y}\right)$ the temporal and spatial gradients at time $t$ (first image). Let call it DOFE_1.

The finite difference of the temporal gradient $I_{t}(\mathbf{s}, t)$ using the two-frame image sequence is:

$$
I_{t}(\mathbf{s}, t)=\frac{I(\mathbf{s}, t+\Delta t)-I(\mathbf{s}, t)}{\Delta t}
$$

From eq, 4 and eq[3. We obtain then a new non-centered DOFE that contains spatial information from both images. We call it TI_DOFE:

$$
\Delta t . I_{t}(\mathbf{s}, t)+\mathbf{d}(\mathbf{s}) \cdot \frac{1}{2}(\nabla I(\mathbf{s}, t)+\nabla I(\mathbf{s}, t+\Delta t)) \approx 0
$$

\subsection{MRF Framework and Minimization}

The displacement field $\mathbf{d}$ is considered as a random variable that maximizes a joint probability. It is computed within a MRF framework via Maximum a Posteriori estimation using a Bayesian decomposition of a Gibbs distribution.

$$
P(\mathbf{d}(\mathbf{s}), I(\mathbf{s}))=\frac{1}{Z} e^{-E(\mathbf{d}(\mathbf{s}), I(\mathbf{s}))}
$$

Where $Z$ is the normalization constant and the total Gibbs energy $\mathrm{E}$ is defined by:

$$
E(\mathbf{d}(\mathbf{s}), I(\mathbf{s}))=\sum_{\mathbf{s} \in C_{1}} V_{d}(\mathbf{d}(\mathbf{s}), I(\mathbf{s}))+\sum_{\mathbf{s}, \mathbf{s}^{\prime} \in C_{2}} \alpha_{p} V_{p}\left(\mathbf{d}(\mathbf{s}), \mathbf{d}\left(\mathbf{s}^{\prime}\right)\right)
$$

$I(\mathbf{s})$ represents the observed data extracted from image intensities. $C_{1}$ and $C_{2}$ are respectively the single-site and pair-site cliques. $\alpha_{p}$ is a weighting coefficient that is used to play on the influence of the data term $V_{d}$ compared to the prior term $V_{p} . V_{p}$ only depends of its 4-neighborhood ( $\mathbf{s}^{\prime}$ neighbor of $\mathbf{s}$ ). 
Data term is a quadratic function of DOFE_1 (eq4), DOFE_2 (eq 3) or TI_DOFE (eq, 66). Eq9 represents the case of TI_DOFE:

$$
V d(\mathbf{d}(\mathbf{s}), I(\mathbf{s}))=\left(\Delta t . I_{t}(\mathbf{s}, t)+\mathbf{d}(\mathbf{s}) \cdot \frac{1}{2}(\nabla I(\mathbf{s}, t)+\nabla I(\mathbf{s}, t+\Delta t))\right)^{2}
$$

DOFE does not admit a unique solution. To solve the ill-posed problem, we add a prior term (regularization) that reduces the configuration of possible solutions. The prior term is defined as Tikhonov regularization [16:

$$
V p\left(\mathbf{d}(\mathbf{s}), \mathbf{d}\left(\mathbf{s}^{\prime}\right)\right)=\left\|\mathbf{d}(\mathbf{s})-\mathbf{d}\left(\mathbf{s}^{\prime}\right)\right\|^{2}
$$

The minimization of the energy is achieved by a Direct Descent Energy (DDE). DDE consists to minimize $E(\mathbf{d}(\mathbf{s}), I(\mathbf{s}))$ by successive iterations over all pixels. A small incremental $\delta \mathbf{d}(\mathbf{s})$ random value is generated where $\mathbf{d}(\mathbf{s}) \leftarrow \mathbf{d}(\mathbf{s})+$ $\delta \mathbf{d}(\mathbf{s}) . \delta \mathbf{d}(\mathbf{s})$ is conserved only if $E(\mathbf{d}(\mathbf{s}), I(\mathbf{s}))$ is decreased. This minimization method converges to a local minimum of the energy. It is then dependent to the initialization of displacement field. To cope with this problem, the weighting coefficient $\alpha_{p}(i)$ is logarithmic increasing over the iteration $(i)$ from 0 to $\alpha_{p}$. In this way, the estimated displacement field satisfies first the DOFE then it is slowly becoming more constrained by the regularization term. Moreover, the multigrid technique allows the DDE minimization to not be trapped into local minima and to reach an optimal solution.

\section{Combined Multiresolution - Multigrid}

\subsection{Pyramidal Decomposition}

The multiresolution by pyramidal decomposition from coarse to fine resolution has been proved to be numerically useful for optical flow estimation 14. The image resolution is iteratively reduced in a pyramid of $K$ different successive resolution levels from the original resolution using Gaussian filter 6]. We use a Gaussian filter of variance $\sigma=1$.

At each pyramid level $k$, the total displacement field $\mathbf{d}^{k}=\tilde{\mathbf{d}}^{k+1}+\mathbf{d}^{\prime k}$ where $\tilde{\mathbf{d}}^{k+1}$ is the interpolated total displacement field computed at coarser resolution $(k+1)$ and $\mathbf{d}^{\prime k}$ is the complementary displacement field at level $k . \mathbf{d}^{\prime k}$ is small at each pyramid level $k$. The $1^{\text {st }}$ order Taylor expansion condition is then respected for each level. The TI_DOFE becomes:

$$
\Delta t \cdot I_{t}\left(\mathbf{s}+\tilde{\mathbf{d}}^{k+1}, t\right)+\mathbf{d}^{\prime k} \cdot \frac{1}{2}\left(\nabla I\left(\mathbf{s}+\tilde{\mathbf{d}}^{k+1}, t\right)+\nabla I\left(\mathbf{s}+\tilde{\mathbf{d}}^{k+1}, t+\Delta t\right)\right) \approx 0
$$

For better readability, we did not write the spatial dependency of the displacement field $\left(\tilde{\mathbf{d}}^{k+1}(\mathbf{s}), \mathbf{d}^{\prime k}(\mathbf{s})\right)$. By similarity, DOFE_1 and DOFE_2 for multiresolution can easily be obtained in the same way.

To compute the observed data of the equation, common methods warp the image $(I)$ into a compensated intermediate image $(\hat{I})$ depending to the used DOFE formulation : [5] warps the first frame to the second $\hat{I}(\mathbf{s}, t)=I\left(\mathbf{s}+\tilde{\mathbf{d}}^{k+1}, t\right)$ 
or 813. warps the second to the first $\hat{I}(\mathbf{s}, t+\Delta t)=I\left(\mathbf{s}+\tilde{\mathbf{d}}^{k+1}, t+\Delta t\right)$. The image $(I)$ is transformed by the displacement field $\tilde{\mathbf{d}}^{k+1}$, then the intensity distribution is interpolated to a regular pixel grid $\mathbf{s}$. Notes that $\mathbf{s}$ in $\hat{I}(\mathbf{s}, t)$ represents in fact the old position $\mathbf{s}+\tilde{\mathbf{d}}^{k+1}$ and $\mathbf{s}$ in $\hat{I}(\mathbf{s}, t+\Delta t)$ still represents the same $\mathbf{s}$.

The multiresolution TI_DOFE with warping (W_MR) can be written as:

$$
\Delta t . \hat{I}_{t}(\mathbf{s}, t)+\mathbf{d}^{\prime k} \cdot \frac{1}{2}(\nabla \hat{I}(\mathbf{s}, t)+\nabla \hat{I}(\mathbf{s}, t+\Delta t)) \approx 0
$$

The spatial and temporal gradients are computed from the warped images. Their precision depends to the quality of $\tilde{\mathbf{d}}^{k+1}$ estimation and to the efficiency of warping technique. $\hat{I}_{t}(\mathbf{s}, t)=I(\mathbf{s}, t+\Delta t)-\hat{I}(\mathbf{s}, t)=\hat{I}(\mathbf{s}, t+\Delta t)-I(\mathbf{s}, t)$.

In this paper, we proposed to suppress the warping step. We consider that the spatial derivatives are locally invariant over a small time interval $\Delta t$. It means that the gradients of DOFE can be computed on both original images. No image needs to be warped. Then we use the correct gradient quantity in respect to the coarse interpolated displacement field $\tilde{\mathbf{d}}^{k+1}(\mathbf{s})$ for each pixel $\mathbf{s}$.

The multiresolution TI_DOFE without warping (noW_MR) take the following form:

$$
\Delta t . I_{t}\left(\mathbf{s}+\tilde{\mathbf{d}}^{k+1}, t\right)+\mathbf{d}^{\prime k} \cdot \frac{1}{2}\left(\nabla I(\mathbf{s}, t)+\nabla I\left(\mathbf{s}+\tilde{\mathbf{d}}^{k+1}, t+\Delta t\right)\right) \approx 0
$$

Where $\nabla I(\mathbf{s}, t)=\nabla I\left(\mathbf{s}+\tilde{\mathbf{d}}^{k+1}, t\right)$ is the spatial gradient on the first image, $\nabla I\left(\mathbf{s}+\tilde{\mathbf{d}}^{k+1}, t+\Delta t\right)$ is the spatial gradient on the second image at coordinates $\mathbf{s}+\tilde{\mathbf{d}}^{k+1}, \Delta t \cdot I_{t}\left(\mathbf{s}+\tilde{\mathbf{d}}^{k+1}, t\right)=I\left(\mathbf{s}+\tilde{\mathbf{d}}^{k+1}, t+\Delta t\right)-I(\mathbf{s}, t)$ is the difference of the intensity at coordinates $\mathbf{s}+\tilde{\mathbf{d}}^{k+1}$ on the second image with the intensity at $\mathbf{s}$ on the first one. We use a bilinear interpolation to compute the corresponding value of $\nabla I\left(\mathbf{s}+\tilde{\mathbf{d}}^{k+1}, t+\Delta t\right)$ and $I\left(\mathbf{s}+\tilde{\mathbf{d}}^{k+1}, t+\Delta t\right)$.

To resume, W_MR warps image information then compute spatial and temporal gradients. In noW_MR, due to local invariant hypothesis on gradients, gradients can be first computed on original images then only values in $\mathbf{s}+\tilde{\mathbf{d}}^{k+1}$ are interpolated.

The prior term along the multiresolution scheme is still the Tikhonov regularization of the total displacement field $\mathbf{d}^{k}(\mathbf{s})$ :

$$
V p\left(\mathbf{d}^{k}(\mathbf{s}), \mathbf{d}^{k}\left(\mathbf{s}^{\prime}\right)\right)=\left\|\left(\tilde{\mathbf{d}}^{k+1}(\mathbf{s})+\mathbf{d}^{\prime k}(\mathbf{s})\right)-\left(\tilde{\mathbf{d}}^{k+1}\left(\mathbf{s}^{\prime}\right)+\mathbf{d}^{\prime k}\left(\mathbf{s}^{\prime}\right)\right)\right\|^{2}
$$

\subsection{Multigrid Method}

At each pyramid level $k$, we use a two-step multigrid method previously proposed in [7. The complementary displacement field $\mathbf{d}^{\prime k}$ is decomposed into a global component $\mathbf{d}_{\mathbf{g}}{ }^{\prime k}$ (average over a mesh size) and a local component $\mathbf{d}_{\mathbf{l}}{ }^{\prime k}$ (local deviation from $\mathbf{d}_{\mathbf{g}}{ }^{\prime k}$ for each pixel). $\mathbf{d}_{\mathbf{g}}{ }^{\prime k}$ is very fast to compute and furnishes a good approximation of the final displacement. It is used to initialize the search of $\mathbf{d}^{\prime k}=\mathbf{d}_{\mathbf{g}}{ }^{\prime k}+\mathbf{d}_{\mathbf{l}}{ }^{\prime k}$ at pixel level. Computational time is faster and the minimization can reach a better solution closer to the optimal. 


\section{Results}

For all the illustrated results, we use the same parameter definitions to be able to compare the DOFE formulations and multiresolution schema. We set a 4-level pyramid decomposition for multiresolution and a grid size of $4 \times 4$ pixels for the multigrid method. The weighting coefficient $\alpha_{p}$ is spatially constant and equal to 100 .

We evaluate the performance of our methods on the recent Middlebury optical flow benchmark dataset [3. We use, in this paper, the Average Angle Error (AAE) [43] criteria to compare the efficiency of estimations:

$$
A E=\arccos \left(\frac{\mathbf{d}_{\mathbf{c}}(\mathbf{s}) \cdot \mathbf{d}_{\mathbf{e}}(\mathbf{s})}{\left\|\mathbf{d}_{\mathbf{c}}(\mathbf{s})\right\|\left\|\mathbf{d}_{\mathbf{e}}(\mathbf{s})\right\|}\right)
$$

$\mathrm{AE}$ is the angle error between the correct displacement $\mathbf{d}_{\mathbf{c}}$ and the estimated displacement $\mathbf{d}_{\mathbf{e}}$. The AAE is computed for three kinds of image area: all the image domain without border (all), the motion discontinuities (disc) and textureless regions (untext). We pre-process the data by convolving each frame of the sequence with a smoothing Gaussian filter $(\sigma=1)$.

We discuss the results of DOFE_1, DOFE_2 and TI_DOFE using W_MR or noW_MR on the dimetrodon sequence. Fig 1 shows the first input image, the ground truth where displacement vectors are coded with the color map proposed in [3] and the estimated displacement vector field computed using the two-frame optical flow formulation (TI_DOFE) with unwarping multiresolution scheme (noW_MR). The second line of Fig 1 illustrates the three masks used to compute AAE for all image domain, motion discontinuity area and textureless regions.

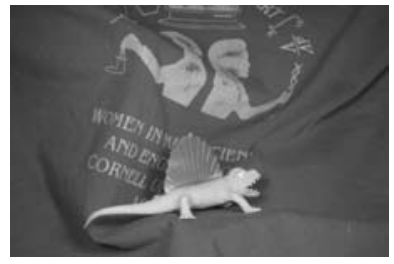

(a) first Image

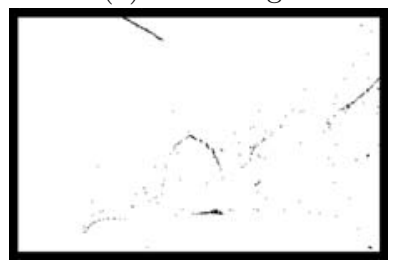

(d) Mask all

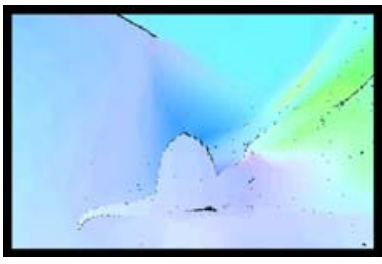

(b) Ground truth

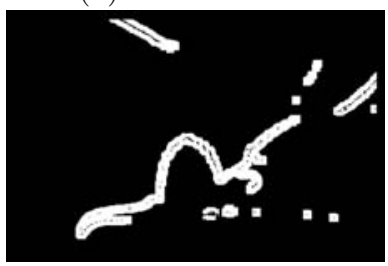

(e) Mask disc

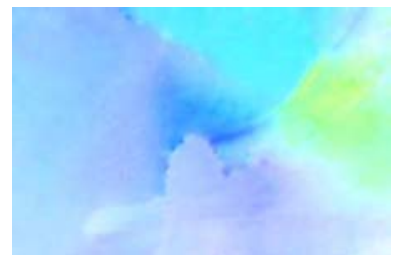

(c) TI_DOFE with noW_MR

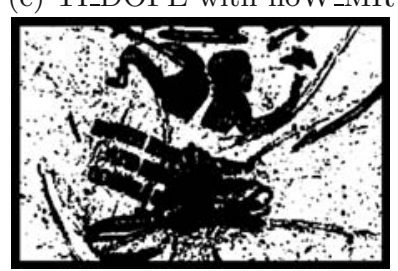

(f) Mask untext

Fig. 1. Dimetrodon: One of the three types of data illustrated in this paper. Ground truth and estimated field (TI_DOFE with noW_MR) are represented by flow field color coding map (3). Only white area is used to compute the AAE for the different masks. 
The estimated field is very similar to the ground truth field. Because it is difficult to visualize the difference between the approaches, table 1 shows the different AAE values for three kinds of sequences (Dimetrodon, Yosemite and Venus) for the different DOFE formulation using warping and unwarping multiresolution. Performance of our methods is also compared to results shown in [3] of few classic optical flow algorithms.

Table 1. AAE comparison of our methods with classic algorithms ( 3 ) for Dimetrodon, Yosemite and Venus sequences. In bold: smallest AAE for classic algorithms, W_MR and noW_MR. In red: smallest AAE over all methods.

\begin{tabular}{|c|ccc|ccc|ccc|}
\hline AAE & \multicolumn{3}{|c|}{ dimetrodon } & \multicolumn{3}{|c|}{ yosemite } & \multicolumn{4}{c|}{ venus } \\
& all & disc & untext & all & disc & untext & all & disc & untext \\
\hline \hline Bruhn et al. & 10.99 & $\mathbf{9 . 4 1}$ & 14.22 & $\mathbf{1 . 6 9}$ & $\mathbf{2 . 8 6}$ & $\mathbf{1 . 0 5}$ & 8.73 & 31.46 & 8.15 \\
Black and Anandan & $\mathbf{9 . 2 6}$ & 10.11 & $\mathbf{1 2 . 0 8}$ & 2.65 & 4.18 & 1.88 & $\mathbf{7 . 6 4}$ & $\mathbf{3 0 . 1 3}$ & $\mathbf{7 . 3 1}$ \\
Pyramid LK & 10.27 & 9.71 & 13.63 & 5.22 & 6.64 & 4.29 & 14.61 & 36.18 & 24.67 \\
MediaPlayer TM & 15.82 & 26.42 & 16.96 & 11.09 & 17.16 & 10.66 & 15.48 & 43.56 & 15.09 \\
Zitnick et al. & 30.10 & 34.27 & 31.58 & 18.50 & 28.00 & 9.41 & 11.42 & 31.46 & 11.12 \\
\hline \hline W_MR DOFE_1 & 5.20 & 8.62 & 6.17 & 3.21 & 4.88 & $\mathbf{1 . 3 3}$ & 8.56 & 34.85 & 8.21 \\
W_MR DOFE_2 & 5.43 & 8.72 & 6.19 & 3.49 & $\mathbf{4 . 7 5}$ & 2.01 & 9.57 & 35.17 & 9.02 \\
W_MR TI_DOFE & $\mathbf{5 . 0 0}$ & $\mathbf{8 . 4 3}$ & $\mathbf{5 . 8 9}$ & $\mathbf{3 . 1 7}$ & 4.81 & 1.35 & $\mathbf{8 . 3 2}$ & $\mathbf{3 4 . 8 1}$ & $\mathbf{7 . 9 0}$ \\
\hline noW_MR DOFE_1 & 5.12 & 8.50 & 6.02 & 2.89 & $\mathbf{4 . 1 3}$ & 1.23 & 9.03 & 35.28 & 8.71 \\
noW_MR DOFE_2 & 4.99 & $\mathbf{8 . 0 9}$ & 5.80 & 2.93 & 4.15 & 1.12 & 8.72 & 34.37 & 8.72 \\
noW_MR TI_DOFE & $\mathbf{4 . 9 2}$ & 8.21 & $\mathbf{5 . 8 0}$ & $\mathbf{2 . 8 8}$ & $\mathbf{4 . 1 3}$ & $\mathbf{1 . 0 6}$ & $\mathbf{8 . 4 1}$ & $\mathbf{3 3 . 8 1}$ & $\mathbf{8 . 5 4}$ \\
\hline
\end{tabular}

From the table, we can remark that our optical flow approach outperforms algorithms as Pyramid LK, MediaPlayer TM and Zitnick and that it gets around the same magnitude of AAE than Bruhn et al. and Black and Anandan. In bold red are the smallest AAE over all methods for each sequence and each method produces at least one of the best estimation.

The optical flow formulation using the two-frame spatial information (TI_DOFE) performs better than optical flow definitions based on only one image information (DOFE_1, DOFE_2) independently to the used multiresolution schema. The new unwarping multiresolution method allows most of the time a better estimation of the displacement field for all kind of optical flow formulations.

However, we can notice that our methods have clearly stronger AAE for motion discontinuity areas. This is due to the MRF formulation of our energy terms that are defined as quadratic functions.

Further results over the all Middlebury optical flow benchmark dataset, including comparisons to other recent techniques are available at the website: http://vision.middlebury.edu/flow/.

\section{Conclusion}

In this work, we propose a two-frame optical flow formulation using the spatial information from the two images. A new unwarping multiresolution scheme is 
defined that reduces the number of transformation and interpolation during the pyramidal decreasing process to estimate the displacement field.

Results have shown that the combination of TI_DOFE and noW_MR methods increases the performance of optical flow estimation. The estimation efficiency is as good as state of the art algorithms. It is interesting in a future work to introduce robust function in our MRF framework to be able to extract better motion discontinuities and to define a better data and prior function that physically correspond to the studied motion phenomenon.

\section{References}

1. Alvarez, L., Castano, C.A., Garcia, M., Krissian, K., Mazorra, L., Salgado, A., Sanchez, J.: Symmetric Optical Flow. In: Moreno Díaz, R., Pichler, F., Quesada Arencibia, A. (eds.) EUROCAST 2007. LNCS, vol. 4739, pp. 676-683. Springer, Heidelberg (2007)

2. Alvarez, L., Weickert, J., Sanchez, J.: Reliable Estimation of Dense Optical Flow Fields with Large Displacements. IJCV 39, 41-56 (2000)

3. Baker, S., Roth, S., Scharstein, D., Black, M.J., Lewis, J.P., Szeliski, R.: A Database and Evaluation Methodology for Optical Flow. ICCV 2007, 1-8 (2007), http://vision.middlebury.edu/flow/

4. Barron, J.L., Fleet, D.J., Beauchemin, S.S.: Performance of Optical Flow Techniques. IJCV 12, 43-77 (1994)

5. Black, M.J., Anandan, P.: The Robust Estimation of Multiple Motions: Parametric and Piecewise-Smooth Flow-Fields. CVIU 63, 75-104 (1996)

6. Burt, P.J., Adelson, E.H.: The Laplacian Pyramid as a Compact Image Code. IEEE Trans. on Communications 3, 532-540 (1983)

7. Cassisa, C., Prinet, V., Shao, L., Simoens, S., Liu, C.L.: Optical flow robust estimation in a hybrid multi-resolution MRF Framework. In: ICASSP 2008, Las Vegas (2008)

8. Corpetti, T., Menin, E., Perez, P.: Dense estimation of fluid flow. IEEE Trans. on Pattern Analysis and Machine Intelligence, 365-380 (2002)

9. Horn, B.K.P., Schunck, B.G.: Determining Optical Flow. Aritifial Intelligence 17, 185-203 (1981)

10. Liu, H., Rosenfeld, A., Chellapa, R.: Two-frame multi-scale optical flow estimation using wavelet decomposition. In: ICASSP 2002, vol. 3 (2002)

11. Memin, E., Perez, P.: Dense Estimation and Object-Based Segmentation of the Optical-Flow with Robust Techniques. Int. J. on IP 7, 703-719 (1998)

12. Memin, E., Perez, P.: A multigrid approach to hierarchical motion estimation. In: ICCV 1998, January 1998, pp. 933-938 (1998)

13. Papenberg, N., Bruhn, A., Brox, T., Didas, S., Weickert, J.: Highly Accurate Optic Flow Computation with Theoretically Justified Warping. IJCV, 141-158 (2006)

14. Papenberg, N., Bruhn, A., Brox, T., Weickert, J.: Numerical Justification for Multiresolution Optical Flow Computation. In: IWCVIA 2003, Las Palmas, Spain (2003)

15. Simoncelli, E.P., Freeman, W.T.: The Steerable Pyramid: A Flexible Architecture for Multi-Scale Derivative Computation. In: ICIP 1995, pp. 444-447 (1995)

16. Tikhonov, A.N., Arsenin, V.Y.: Solutions of ill-posed Problems, ch. 2. John Wiley \& Sons, Chichester (1977) 